\title{
Loss of Smad4 expression predicts liver metastasis in human colorectal cancer
}

\author{
LORENA LOSI ${ }^{1}$, HANIFA BOUZOURENE ${ }^{2}$ and JEAN BENHATTAR ${ }^{2}$ \\ ${ }^{1}$ Department of Pathology, University of Modena and Reggio Emilia, Modena, Italy; \\ ${ }^{2}$ Institute of Pathology, Centre Hospitalier Universitaire Vaudois, Lausanne, Switzerland
}

Received December 1, 2006; Accepted January 18, 2007

\begin{abstract}
Distant metastases represent the major cause of death after curative surgery of colorectal cancer. The aim of this study was to evaluate the role of Smad4 and KRAS genetic alterations in colorectal metastases taking into account both the site (hepatic versus extrahepatic) and the time (synchronous versus metachronous) of recurrence. We examined the immunohistochemical expression of Smad4 and frequency of KRAS mutation in primary colorectal tumors and in their corresponding metastatic tissues. Loss of Smad4 expression was noted in $37 \%(26 / 71)$ of the primary tumors and the corresponding metastases. Absence of Smad4 protein was more frequently observed in hepatic metastases, whether they were metachronous or synchronous, than in extrahepatic metastases $(\mathrm{p}<0.005)$. The frequency of KRAS mutations was high in the synchronous and extrahepatic metachronous metastases $(68-80 \%)$, but was significantly lower in the hepatic metachronous metastases (11\%). Our results indicate that absence of Smad4 expression correlated significantly with liver metastases regardless of the time of their occurrence and represents a promising new biomarker to predict liver metastasis in colorectal cancer patients. Therefore, this group of patients could benefit from a specific and appropriate pre- and/or post-operative therapy.
\end{abstract}

\section{Introduction}

Distant metastases, by hematogenous or to a lesser extent by lymphatic vessels, represent the major cause of death after curative surgery of colorectal cancer (CRC). Liver is the most common site of CRC metastases compared to other organs such as lung, brain or bone (1). Hepatic metastases are present in $30-50 \%$ of patients, and liver is frequently the sole organ harboring metastases. To improve the prognosis of

Correspondence to: Dr Jean Benhattar, Institute of Pathology, CHUV, Bugnon 25, 1011 Lausanne, Switzerland

E-mail: jean.benhattar@chuv.ch

Key words: colorectal cancer, Smad4, KRAS, liver metastases, immunohistochemistry
CRC, the most important considerations are the selection of high-risk patients to administer the appropriate therapy.

Several studies have attempted to find genetic markers that give accurate information on the prognostic impact for patient with CRC. Among these markers, KRAS and Smad4 (DPC4) have been extensively investigated. Oncogenic mutation in KRAS is one of the most common genetic alterations in colorectal cancer and considered an early event in colorectal tumorigenesis (2). Regarding the prognostic significance of KRAS gene mutations in colorectal cancer, there is a lack of agreement on how mutations relate to clinical factors. Some groups have suggested that the presence of any KRAS mutation conveys prognostic significance (3-6), whereas others have reached contrary conclusions (7-9).

Smad4 is expressed ubiquitously in different human organ systems. The gene product is an important cellular mediator of TGF- $\beta$ signals relevant for development and control of cell growth. Smad4 forms a complex with Smad2 and Smad3, and translocates to the nucleus where it activates transcription of multiple TGF- $\beta$ response genes (10). Smad4 mutations have been shown to be associated with the occurrence of juvenile polyposis (11) and frequent somatic mutations have been found as a late event in colorectal cancers, further suggesting an important role for this gene in colorectal carcinogenesis $(12,13)$. Many studies showed that Smad4 alterations were more frequent in advanced colorectal disease and in metastatic cancers (13-18).

Some genetic abnormalities could be specifically required for the development of metastatic tumor cells in function of the recipient organ. In a recent study, we analyzed colorectal metachronous metastases and we found a good correlation between some of the genetic alterations identified in the tumor cells and the location of the metastases (19). However, the precise mechanism for the homing of colorectal metastases and the selected metastatic organ remains largely unknown.

In the present study, we examined the frequency of Smad4 and KRAS alterations in different groups of patients with CRC and developing metastases. We verified the role of these genetic changes in the different metastatic processes taking into account the mode of recurrence, regarding both the site (hepatic versus extrahepatic) and the time (synchronous versus metachronous), with the aim to select appropriate biomarkers useful for predicting metastases from colorectal cancers. 
Table I. Clinicopathological characteristics of patients who developed metastases after radical curative surgery for colorectal cancer or metastases present at the time of surgery.

\begin{tabular}{|c|c|c|c|c|c|}
\hline \multirow[b]{2}{*}{ Characteristics } & \multicolumn{2}{|c|}{ Metachronous metastases } & \multicolumn{2}{|c|}{ Synchronous metastases } & \multirow[b]{2}{*}{ Total } \\
\hline & $\begin{array}{c}\text { Extrahepatic } \\
\text { (Group 1) }\end{array}$ & $\begin{array}{l}\text { Hepatic } \\
\text { (Group 2) }\end{array}$ & $\begin{array}{l}\text { Extrahepatic } \\
\text { (Group 3) }\end{array}$ & $\begin{array}{l}\text { Hepatic } \\
\text { (Group 4) }\end{array}$ & \\
\hline \multicolumn{6}{|l|}{ Gender } \\
\hline Male & 10 & 11 & 5 & 13 & 39 \\
\hline Female & 10 & 8 & 5 & 9 & 32 \\
\hline Age & $46-81(61.4)$ & $50-84(62)$ & $46-77(65.7)$ & $51-80(64.9)$ & $46-84$ \\
\hline \multicolumn{6}{|l|}{ Tumor location } \\
\hline Proximal & 4 & 4 & 1 & 8 & 17 \\
\hline Distal & 15 & 11 & 6 & 12 & 44 \\
\hline Rectum & 1 & 4 & 3 & 2 & 10 \\
\hline \multicolumn{6}{|l|}{ Differentiation } \\
\hline Well & 4 & 4 & 1 & 2 & 11 \\
\hline Moderate & 15 & 11 & 7 & 17 & 50 \\
\hline Poor & 1 & 4 & 2 & 3 & 10 \\
\hline \multicolumn{6}{|l|}{ Dukes' stage } \\
\hline B & 11 & 7 & 0 & 0 & 18 \\
\hline $\mathrm{C}$ & 9 & 12 & 0 & 0 & 21 \\
\hline $\mathrm{D}$ & 0 & 0 & 10 & 22 & 32 \\
\hline \multicolumn{6}{|c|}{ Adjuvant therapy } \\
\hline Yes & 7 & 7 & 8 & 21 & 43 \\
\hline No & 13 & 12 & 2 & 1 & 28 \\
\hline
\end{tabular}

\section{Materials and methods}

Patients and tissue specimens. The surgical specimens were obtained from a total of 91 patients who were treated surgically for primary sporadic CRC between 1993 to 2002. The cases were selected from the Pathology Department of the University of Modena and Reggio Emilia and from the Institute of Pathology of Lausanne. Fourteen patients received adjuvant post-operative 5-FU chemotherapy. Seventy-one patients developed metastases after (Groups 1 and 2) or at the time (Groups 3 and 4) of surgery.

Group 1: 20 patients with distant extrahepatic metastases resected 4-79 months after the primary tumor. The analyzed metastases were from the lung (14), the stomach (1), the thyroid (1) and the bone (4).

Group 2: 19 patients with distant liver metastases resected 4-51 months after the primary tumor.

Group 3: 10 patients with extrahepatic distant metastases to lung (6), bone (2) or brain (2), resected at the time of surgery.

Group 4: 22 patients with distant liver metastases resected at the time of surgery.

Group 5: 20 CRC patients with stage B and C. This group consists of patients disease-free at a 5-years follow-up obtained through direct contact, clinical charts and pathological records. There were 10 men and 10 women with a mean age of 64.4 years (range 45-79). The tumor was localized in the proximal colon in 6 cases, distal colon in 9 cases and rectum in 5 cases.

Single-strand conformation polymorphism (SSCP) analysis of the KRAS gene. Genomic DNA was extracted from fixed materials using the DNeasy tissue kit (Qiagen, Hilden, Germany). The DNA was eluted from the column with $60 \mu 1$ of elution buffer and kept at $-20^{\circ} \mathrm{C}$ until use. Exon 1 of the KRAS gene was amplified by PCR and mutation were detected by SSCP, as previously described (19).

Immunohistochemistry. Sections ( $4 \mu \mathrm{m})$ were cut from paraffin blocks and deparaffinized using standard methods. Slides were treated with sodium citrate and steamed for $30 \mathrm{~min}$ at $80^{\circ} \mathrm{C}$. After cooling for $5 \mathrm{~min}$, the slides were labeled with a monoclonal antibody specific to Smad4 (clone B8, Santa Cruz Biotechnology, Santa Cruz, CA) at a dilution of 1:100. The antibody was detected using a biotinylated secondary antibody and 3,3'-diaminobenzidine as the chromogen. Then, the slides were hematoxylin counterstained.

For Smad4, positive labeling was defined as strong to moderate staining of the cytoplasm of cells, with focal expression in the nuclei. Absence of expression was interpreted as negative. Alveolar and bronchiolar cells in the lung, hepatocytes in the liver, fibroblasts and lymphocytes, all showing moderate to strong expression of the Smad4 gene, served as 


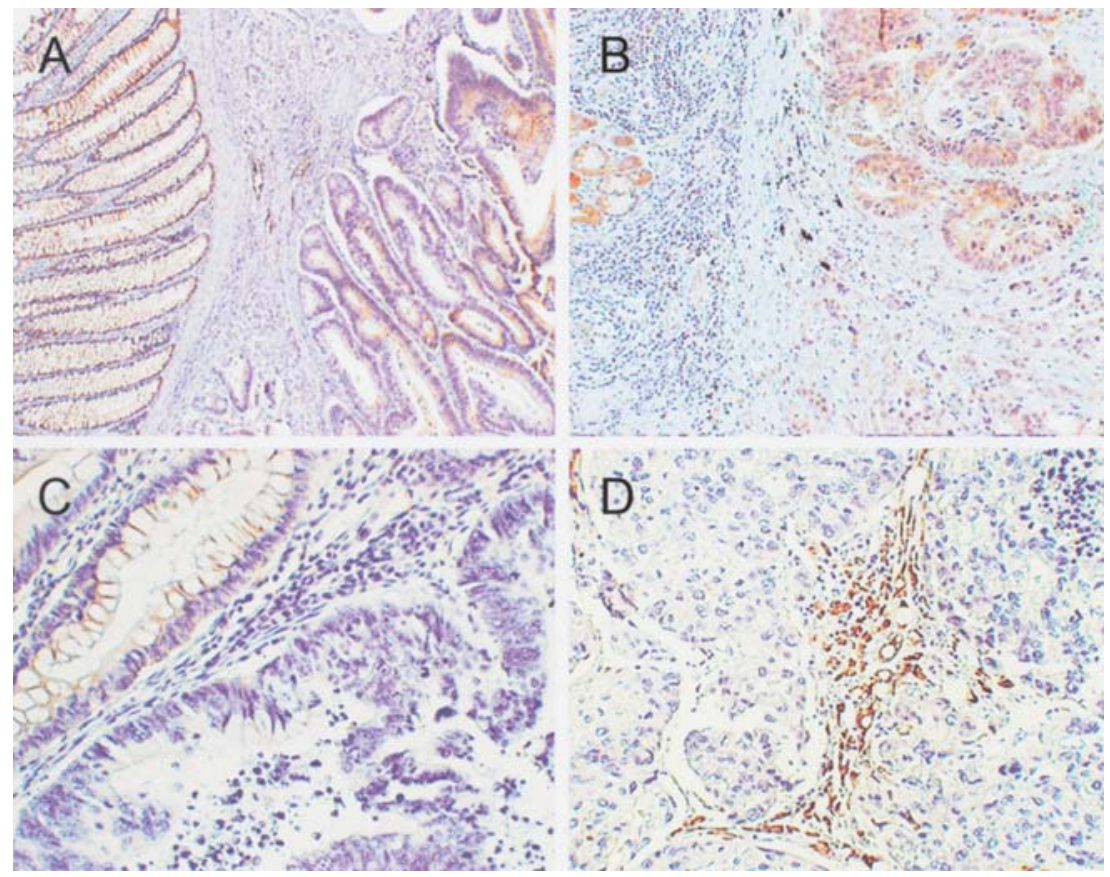

Figure 1. Smad4 immunohistochemistry performed on primary CRC (A and C) and their corresponding metastases (B and D). Positive cytoplasmic expression of Smad4 observed in a primary CRC and its corresponding lung metastasis (A and B, original magnification x10). In another case, loss of Smad4 expression can be noted in the primary CRC and in the liver metastasis (C and D, original magnification $\mathrm{x} 20)$.

Table II. Genetic alterations in metastases developed after radical curative surgery for colorectal cancer (metachronous metastases) or at the time of surgery (synchronous metastases).

\begin{tabular}{lllllll}
\hline & \multicolumn{2}{c}{ Metachronous metastases } & & \multicolumn{2}{c}{ Synchronous metastases } & \\
\cline { 2 - 3 } & $\begin{array}{l}\text { Extrahepatic } \\
\text { (Group 1) }\end{array}$ & $\begin{array}{c}\text { Hepatic } \\
\text { (Group 2) }\end{array}$ & & $\begin{array}{c}\text { Extrahepatic } \\
\text { (Group 3) }\end{array}$ & $\begin{array}{c}\text { Hepatic } \\
\text { (Group 4) }\end{array}$ & Total \\
\hline KRAS mutation & $75 \%(15 / 20)$ & $11 \%(2 / 19)$ & & $80 \%(8 / 10)$ & $68 \%(15 / 22)$ & $56 \%(40 / 71)$ \\
Loss of Smad4 & $15 \%(3 / 20)$ & $53 \%(10 / 19)$ & & $10 \%(1 / 10)$ & $55 \%(12 / 22)$ & $37 \%(26 / 71)$ \\
\hline
\end{tabular}

internal positive controls. The primary antibody was omitted in the negative controls.

Statistical analysis. The Fisher's exact test was used to evaluate the association between two dichotomous variables. A p-value of $<0.05$ was considered to indicate statistical significance.

\section{Results}

The clinical and pathological data of the 71 colorectal cancer patients, having developed either metachronous or synchronous metastasis, are summarized in Table I.

The expression of Smad4 was similar in the primary tumors and the corresponding metastases. Loss of Smad4 expression was noted in $37 \%(26 / 71)$ of the cases. Fig. 1 shows immunohistochemistry of Smad4 with either positive or negative cytoplasmic staining of primary tumor cells and corresponding metastatic tumor cells. Absence of Smad4 was more frequently observed in hepatic metastases, whether they were metachronous or synchronous (53 and
$55 \%$, respectively), than in metachronous and synchronous extrahepatic metastases (15 and 10\%, respectively) (Table II). The difference was statistically significant $(\mathrm{p}<0.005)$. In the comparative group of 20 non-metastatic patients, Smad4 expression was absent in only 3 tumors $(15 \%)$, an occurrence similar to those observed in the extrahepatic metastatic group.

SSCP analysis revealed 40 of $71(56 \%)$ cases with shifted bands for the KRAS exon 1 . In all but one case, the same mutation was identified in the primary tumor and in the corresponding metastasis. Mutations occurred preferentially at the second base of codon $12(27 / 40,68 \%)$ and were GGT to GTT or GAT or GCT, in 17, 6 and 4 cases, respectively. Other mutations were observed at the first base of codon 12 in 6 cases and at the second base of codon 13 (GGC to GAC) in the last 7 cases. KRAS mutation occurred at a high level in the extrahepatic synchronous and metachronous metastases (80 and $75 \%$, respectively), as well as in hepatic synchronous metastases $(68 \%)$. On the contrary, the level of KRAS mutation was significantly lower in hepatic metachronous metastases $(11 \%, 2 / 19$ cases $)(p<0.0001)$ (Table II). 


\section{Discussion}

In the present study, we investigated the association of Smad4 and KRAS alterations with the location (hepatic versus extrahepatic) and the time of recurrence (synchronous versus metachronous) of distant metastasis with the aim to identify powerful biomarkers for predicting metastasis of colorectal cancers. This could permit the selection of patients who may be candidates for an appropriate chemotherapy pre- and/or postoperative.

Loss of Smad4 expression was more frequently observed in synchronous and metachronous liver metastases than in other distant metastases. The expression of Smad4 was similar in the primary CRC and the corresponding metastases. As observed in tumors with extrahepatic metastases, loss of Smad4 expression was also uncommon in the primary CRC without distant metastases. Therefore, absence of Smad4 expression in the primary colorectal cancer cells was significantly correlated with liver metastasis occurrence.

Smad4 is a central component of the TGF- $/$ /Smad pathway. Deletion or degradation of Smad4 in tumors could specifically inhibit the tumor suppressor effect of TGF- $\beta$ (20). Smad4 alteration has been associated with specific loss of TGF- $3-$ induced growth resulting in increased angiogenesis and loss of epithelial integrity $(21,22)$. Studies analyzing cancers at different stages show Smad4 inactivation to be associated with the advanced disease state in colorectal tumorigenesis $(13,14)$. Our results confirm that Smad4 is downregulated during tumor progression. Nevertheless, many studies that evaluated the prognostic value of Smad4 expression using immonohistochemical techniques have found Smad4 expression decreased in metastatic cancers, but did not take into account the mode of recurrence $(14,16,17)$. In our study, we focused our attention on the different types of metastasis regarding both the site and the time of recurrence. According to our data, Smad4 was associated with the occurrence of liver metastasis in CRC patients regardless of the time of the recurrence, suggesting that loss of Smad4 may specifically contribute to the establishment of colorectal cancer liver metastasis.

Metastasis consists of a series of sequential steps that include the shedding of cells from a primary tumor into the circulation, survival of the cells in the circulation, arrest in a new organ, extravasation into the surrounding tissue, initiation and maintenance of growth, and vascularization of the metastatic tumor (23). Proteome analyses have detected Smad4 targets relevant for tumor-stroma interactions; the Smad4-negative cells produced a major group of proteins of the extracellular matrix, characterized as anti-adhesive and invasion-promoting proteins (24). The stroma microenvironment of target organ for metastasis has the ability to produce adhesion molecules, integrins, stromal metalloproteinases and angiogenic molecules that facilitate the anchorage of tumor cells and promote tumor growth and angiogenesis $(25,26)$. It has been observed that alteration of TGF- $\beta$, an angiostatic molecule, may increase vascularization of metastases and thus, promoting their growth (27). Therefore, we hypothesized that Smad4, through the TGF-ß/Smad pathway, could play a role either when the cancer cells leave the circulation to extravasate into the liver tissue or for the growing of liver micrometastases. Further studies will be necessary to understand why neoplastic colon cells with altered Smad4 expression represent a population that is able to adapt and develop with a relatively high specificity in the liver.

Our study reported a high frequency of KRAS mutation in metachronous and synchronous extrahepatic metastases (75 and $80 \%$, respectively), mainly in the lung; whereas the liver metastases showed KRAS mutated prevalently in the synchronous ones (68\%). KRAS, at least as a biomarker, seems to play a main part in the metastatic process through blood circulation and thus, would be a good indicator of the potential aggressiveness of colorectal tumor cells $(19,28)$. However, we found a small group (11\%) of metachronous liver metastases with KRAS mutation suggesting that KRAS alteration is not mandatory for the development of hepatic metastases or at least of late metastases, and that Smad4 or other genes most likely drive the mechanism of metachronous hepatic metastases.

In conclusion, our data raise the possibility that absence of Smad4 expression, alone or in combination with other conventional pathological factors, could be a surrogate marker for liver metastasis after curative resection of Dukes' B and C CRCs. Altered expression of Smad4, and perhaps other members of the TGF-ß/Smad pathway, in biopsy samples of colorectal cancer may provide clinicians useful information to identify patients with high-risk of liver metastasis for initiating a targeted adjuvant therapy, immediately after or even before surgical treatment of the primary tumor. A large-scaled immunohistochemical study, as well as the analysis of the other genes implicated in the TGF- $3 /$ Smad pathway, will be needed before a definite conclusion can be drawn.

\section{Acknowledgements}

We wish to thank Charlotte Bricod, Paola Manni and Luca Fabbiani for their excellent technical assistance. This study was supported in part by the Progetti di ricerca avanzata (Italy).

\section{References}

1. Sadahiro S, Suzuki T, Ishikawa K, Nakamura T, Tanaka Y, Masuda T, Mukoyama S, Yasuda S, Tajima T, Makuuchi H and Murayama C: Recurrence patterns after curative resection of colorectal cancer in patients followed for a minimum of ten years. Hepatogastroenterology 50: 1362-1366, 2003.

2. Fearon ER and Vogelstein B: A genetic model for colorectal tumorigenesis. Cell 61: 759-767, 1990.

3. Benhattar J, Losi L, Chaubert P, Givel JC and Costa J: Prognostic significance of K-ras mutations in colorectal carcinoma. Gastroenterology 104: 1044-1048, 1993.

4. Andreyev HJ, Norman AR, Cunningham D, Oates JR and Clarke PA: Kirsten ras mutations in patients with colorectal cancer: the multicenter 'RASCAL' study. J Natl Cancer Inst 90: 675-684, 1998.

5. Andreyev HJ, Norman AR, Cunningham D, et al: Kirsten ras mutations in patients with colorectal cancer: the 'RASCAL II' study. Br J Cancer 85: 692-696, 2001.

6. Conlin A, Smith G, Carey FA, Wolf CR and Steele RJ: The prognostic significance of K-ras, p53, and APC mutations in colorectal carcinoma. Gut 54: 1283-1286, 2005.

7. Morrin M, Kelly M, Barrett N and Delaney P: Mutations of Kiras and p53 genes in colorectal cancer and their prognostic significance. Gut 35: 1627-1631, 1994. 
8. Samowitz WS, Curtin K, Schaffer D, Robertson M, Leppert M and Slattery ML: Relationship of Ki-ras mutations in colon cancers to tumor location, stage, and survival: a populationbased study. Cancer Epidemiol Biomarkers Prev 9: 1193-1197, 2000.

9. Pan ZZ, Wan DS, Chen G, Li LR, Lu ZH and Huang BJ: Comutation of $\mathrm{p} 53, \mathrm{~K}$-ras genes and accumulation of $\mathrm{p} 53$ protein and its correlation to clinicopathological features in rectal cancer. World J Gastroenterol 10: 3688-3690, 2004.

10. Heldin $\mathrm{CH}$, Miyazono K and ten Dijke P: TGF- $\beta$ signaling from cell membrane to nucleus through SMAD proteins. Nature 390: 465-471, 1997.

11. Howe JR, Roth S, Ringold JC, Summers RW, Jarvinen HJ, Sistonen P, Tomlinson IP, Houlston RS, Bevan S, Mitros FA, Stone EM and Aaltonen LA: Mutations in the Smad4/DPC4 gene in juvenile polyposis. Science 280: 1086-1088, 1998.

12. Koyama M, Ito M, Nagai H, Emi M and Moriyama Y: Inactivation of both alleles of the DPC4/SMAD4 gene in advanced colorectal cancers: identification of seven novel somatic mutations in tumors from Japanese patients. Mutat Res 406: 71-77, 1999.

13. Miyaki M, Iijima T, Konishi M, Sakai K, Ishii A, Yasuno M, Hishima T, Koike M, Shitara N, Iwama T, Utsunomiya J, Kuroki T and Mori T: Higher frequency of Smad4 gene mutation in human colorectal cancer with distant metastasis. Oncogene 20: 3098-3103, 1999

14. Maitra A, Molberg K, Albores-Saavedra J and Lindberg G: Loss of Dpc4 expression in colonic adenocarcinomas correlates with the presence of metastatic disease. Am J Pathol 157: 1105-1111, 2000.

15. Ohtaki N, Yamaguchi A, Goi T, Fukaya T, Takeuchi K, Katayama K, Hirose K and Urano T: Somatic alterations of the DPC4 and Madr2 genes in colorectal cancers and relationship to metastasis. Int J Oncol 18: 265-270, 2001.

16. Xie W, Rimm DL, Lin Y, Shih WJ and Reiss M: Loss of Smad signaling in human colorectal cancer is associated with advanced disease and poor prognosis. Cancer J 9: 302-312, 2003.

17. Alazzouzi H, Alhopuro P, Salovaara R, Sammalkorpi H, Jarvinen H, Mecklin JP, Hemminki A, Schwartz S Jr, Aaltonen LA and Arango D: SMAD4 as a prognostic marker in colorectal cancer. Clin Cancer Res 11: 2606-2611, 2005.

18. Alhopuro P, Alazzouzi H, Sammalkorpi H, Davalos V, Salovaara R, Hemminki A, Jarvinen H, Mecklin JP, Aaltonen LA, Schwartz S Jr and Arango D: Smad4 levels and response to 5-fluorouracil in colorectal cancer. Clin Cancer Res 11: 6311-6316, 2005.
19. Losi L, Luppi G and Benhattar J: Assessment of K-ras, Smad4 and p53 gene alterations in colorectal metastases and their role in the metastatic process. Oncol Rep 12: 1221-1225, 2004.

20. Levy L and Hill CS: Smad4 dependency defines two classes of transforming growth factor $\beta(\mathrm{TGF}-\beta)$ target genes and distinguishes TGF-B-induced epithelial-mesenchymal transition from its antiproliferative and migratory responses. Mol Cell Biol 25: 8108-8125, 2005.

21. Miyazono K, Suzuki H and Imamura T: Regulation of TGF-beta signaling and its roles in progression of tumors. Cancer Sci 94: 230-234, 2003.

22. Elliott RL and Blobe GC: Role of transforming growth factor Beta in human cancer. J Clin Oncol 23: 2078-2093, 2005.

23. Chambers AF, Groom AC and MacDonald IC: Dissemination and growth of cancer cells in metastatic sites. Nat Rev Cancer 2: 563-572, 2002.

24. Volmer MW, Radacz Y, Hahn SA, Klein-Scory S, Stuhler K, Zapatka M, Schmiegel W, Meyer HE and Schwarte-Waldhoff I: Tumor suppressor Smad4 mediates downregulation of the antiadhesive invasion-promoting matricellular protein SPARC: Landscaping activity of Smad4 as revealed by a 'secretome' analysis. Proteomics 4: 1324-1334, 2004.

25. Gassmann P, Enns A and Haier J: Role of tumor cell adhesion and migration in organ-specific metastasis formation. Onkologie 27: 577-582, 2004

26. Donadio AC, Durand S, Remedi MM, Frede S, Ceschin DG, Genti-Raimondi S and Chiabrando GA: Evaluation of stromal metalloproteinases and vascular endothelial growth factors in a spontaneous metastasis model. Exp Mol Pathol 79: 259-264, 2005.

27. Tu WH, Thomas TZ, Masumori N, Bhowmick NA, Gorska AE, Shyr Y, Kasper S, Case T, Roberts RL, Shappell SB, Moses HL and Matusik RJ: The loss of TGF- $\beta$ signaling promotes prostate cancer metastasis. Neoplasia 5: 267-277, 2003.

28. Castagnola P and Giaretti W: Mutant KRAS, chromosomal instability and prognosis in colorectal cancer. Biochim Biophys Acta 1756: 115-125, 2005. 